\title{
Effect of resonances on the transport properties of two-dimensional disordered systems
}

\author{
Brahim Elattari ${ }^{1,2}$, Tsampikos Kottos ${ }^{3}$ \\ ${ }^{1}$ Departamento de Física Teórica de la Materia Condensada, Universidad Autónoma de Madrid, \\ 28049 Madrid, Spain. \\ ${ }^{2}$ Université Chouaïb Doukkali, Fauclté des Sciences, El Jadida, Morocco \\ ${ }^{3}$ Max-Planck-Institut für Strömungsforschung \\ 37073 Göttingen, Germany
}

\begin{abstract}
We study both analytically and numerically how the electronic structure and the transport properties of a two-dimensional disordered system are modified in the presence of resonances. The energy dependence of the density of states and the localization length at different resonance energies and strengths of coupling between resonances and random states are determined. The results show, that at energy equals to the resonance energy there is an enhancement in the density of states. In contrast, the localization length remains unaffected from the presence of the resonances and is similar to the one of the standard Anderson model. Finally, we calculate the diffusion constant as a function of energy and we reveal interesting analogies with experimental results on light scattering in the presence of Mie resonances.
\end{abstract}

PACS: 71.55.Jv, 72.15.Rn

The study of waves propagating in disordered lattices, during the past years has attracted much attention and many interesting phenomena have been, more or less, well understood. However, until recently the interest of the physics community was primarily focusing on quantum, i.e. electronic waves. It was the observation of the coherent backscattering effect in classical wave systems [1], the analogous effect to weak localization in the electronic case, which triggered a burst of interest in further studies of scattering disordered classical wave systems. The question of localization of classical waves has attracted attention for two reasons. First, the properties of classical waves such as light waves, microwaves, and acoustic waves in random media are of fundamental interest for their own sake. Second, classical waves can serve as a model system for testing the theory of Anderson localization of electrons experimentally in a clean way, without the complication of strong inelastic scattering and other effects of electron- electron and electron-phonon interaction. Existing theories predict the localization of classical waves under certain circumstances [1] leading thus to the conclusion that the analogy between quantum and classical waves works reasonably well. However, there is no conclusive experimental evidence, and thus the full correspondence is not yet established beyond any doubt (a thorough discussion may be found in Refs. [2. 3]).

The present paper has been motivated by an experimental work, performed several years ago 惯] on the scattering of light by a disordered medium in the presence of Mie resonances. A low concentration of Mie resonances leads to a strong change in the transport properties of the system, reflected in a strong reduction of the transport velocity, or equivalently, of the diffusion constant $D(E)$, near the resonance energies. With increasing concentration of resonant scatterers, the dip in $D(E)$ widens and becomes less deep. Qualitatively speaking, the transport velocity is reduced because a lot of energy is temporar- ily stored inside the resonance or equivalently the wave spends a lot of time (dwell time) inside the Mie resonances.

To understand this surprising result, different theoretical approaches based on different considerations were developed [ [4, 河, [6]. However, there are controversies as to whether this can be applied to the quantum case, i.e., electronic waves. In fact, at low concentration of Mie resonances 泊, in the spirit of the coherent-potential approximation (CPA) 1], it was shown that, unlike electronic systems, the diffusion constant $D(E)$ for classical waves decreases sharply close to the resonance energies. This result was viewed as due to the different Ward identity caused by an energy-dependent scattering potential in the classical case. An extension of the CPA to the strong concentration limit [5], the so called coated CPA, shows that the effect of the Mie resonances decreases at this regime. On the other hand, in a recent work [6], it was shown that an extension of the Random Matrix Theory (RMT) [7], capable of accounting for the presence of resonance scattering, is able to explain the obtained experimental result and generalize it to both quantum and classical waves, although the corresponding Ward identities are different [8].

In the present paper, we extend our previous analysis on the effect of resonances on electrons in a onedimensional disordered medium [9], to higher dimensions. The quantities that will monopolize our interest, will be the density of states (DOS) $\rho(E)$ and the localization length $l_{\infty}(E)$. In the one-dimensional case [9], at low concentration and weak coupling both these quantities are affected strongly by the presence of resonances. The DOS exhibits a Lorentzian peak at the resonance energy and the localization length is drastically decreased except at the resonance energy which remains essentially the same with the one corresponding to the standard Anderson model. We will show that in higher dimensions, the 
density of states exhibits the same resonant enhancement while the localization length remains unaffected in contrast to the one-dimensional case. Finally, by making use of the well known results for quasi-one-dimensional systems we will compute the diffusion constant $D(E)$ and identify similarities with the corresponding problem of light scattering in the presence of Mie resonances.

The mathematical model we consider is the tightbinding Anderson Hamiltonian on a two-dimensional lattice,

$$
H=\sum_{l} \epsilon_{l}|l><l|+\sum_{l, l^{\prime}} t_{l l^{\prime}}\left|l><l^{\prime}\right|
$$

where $(l)$ denote the sites of a two-dimensional lattice $L \times M$. The local site energies $\epsilon_{l}$ are taken independently at random within the interval $[-W / 2, W / 2]$. At some randomly chosen sites $k,(m$ in total), the energies $\epsilon_{k}$ are taken to be fixed i.e. $\epsilon_{k}=E_{r}$. These energies correspond to the resonance energies. To simplify the problem, we assume that all resonances have the same energy $E_{r}$. The hopping matrix elements $t_{l l^{\prime}}$ are restricted to nearest neighbors. When the coupling is between random states ( $N$ in total), $t_{l l^{\prime}}$ are constant taken to be the unit of energy while the coupling between a resonant state and another state $t_{l l^{\prime}}$ has a different constant value $v$.

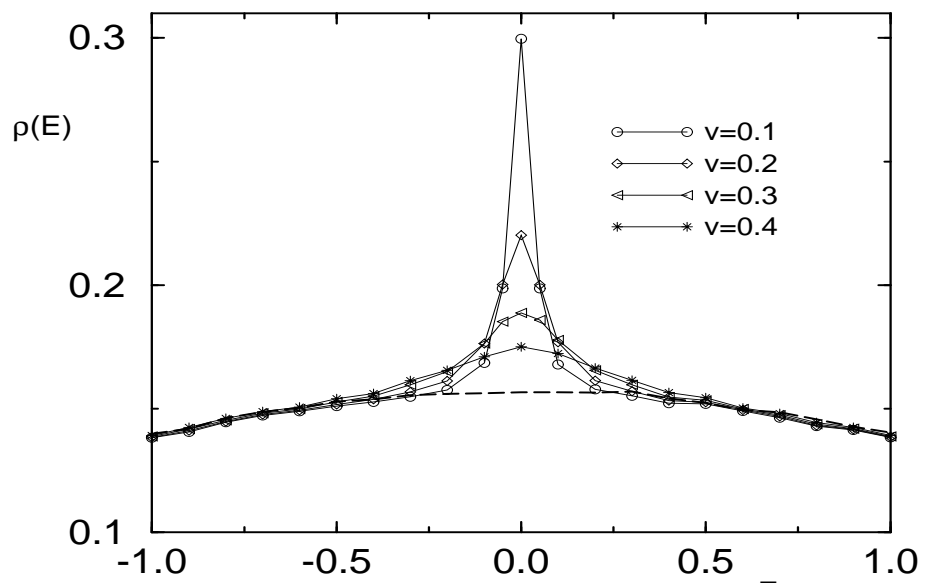

FIG. 1. The density of states $\rho(E)$ for the $2 \mathrm{~d}-$ Anders 6 model in the presence of resonance states with energy $E_{r}=0$ and various values of the coupling constant $v$ corresponding to different symbols. The thick dashed line corresponds to the Anderson model without resonances. In all cases the disorder strength is $W=4$.

To evaluate the density of states $\rho(E)$ of this system, we start by writing the original $(N+m) \times(N+m)$ Hamiltonian matrix $H$ in the following form

$$
H=\left(\begin{array}{cc}
H_{0} & V \\
V^{T} & E_{r} \times I_{m}
\end{array}\right) .
$$

This form can be obtained easily by separating the resonant states from random states. $V^{T}$ denotes the transpose of $V$ and $H_{0}$ is a $N$-dimensional matrix that describes the part of the Hamiltonian $H$ without resonances. Here $I_{m}$ is the $m$-dimensional unit matrix, and $E_{r}$ is the resonance energy of each of the $m$ resonant scatterers. The rectangular matrix $V$ couples the $m$ resonances to $H_{0}$. All the matrix elements of each column of $V$ are zero except four with value $v$.

The Green's function corresponding to the Hamiltonian (21), can be written as

$$
\begin{aligned}
G & =\left(\begin{array}{cc}
G_{0} & G_{V} \\
G_{V}^{T} & G_{r}
\end{array}\right) \\
& =\left(\begin{array}{cc}
E+i \eta-H_{0} & -V \\
-V^{T} & \left(E-E_{r}\right) \times I_{m}+i \eta
\end{array}\right)^{-1} .
\end{aligned}
$$

To determine the different matrices in $G$, we use the previous relation to obtain the following set of equations:

$$
\begin{gathered}
\left(E-H_{0}+i \eta\right) G_{V}-V G_{r}=0, \\
-V^{T} G_{V}+\left[\left(E-E_{r}\right) I+i \eta\right] G_{r}=1 .
\end{gathered}
$$

Solving (4) with respect to $G_{r}$ we get the expression

$$
G_{r}=\frac{1}{\left(E-E_{r}\right) I+i \eta-V^{T} G_{a} V}
$$

where $G_{a}=\frac{1}{E-H_{0}+i \eta}$. In the limit of weak coupling $v \ll 1$ and low concentration of resonances one can make the approximation $G_{a} \simeq G_{0}$. Then the density of states is given by

$$
\rho(E)=-\frac{1}{i \pi}\left(\operatorname{Tr} G_{0}+\operatorname{Tr} \frac{1}{\left(E-E_{r}\right) I+i \eta-V^{T} G_{0} V}\right)
$$

which, in the limit of $v \ll 1$ and low concentration of resonances, can be written as

$$
\rho(E)=\rho_{0}(E)+m \frac{\Gamma / 2 \pi}{\left(E-E_{r}\right)^{2}+\left(\frac{\Gamma}{2}\right)^{2}},
$$

where $\rho_{0}(E)=-\frac{1}{i \pi} \operatorname{Tr} G_{0}$. To a good approximation, $\rho_{0}(E)$ can be identified as the density of states of the standard two-dimensional Anderson model without resonances. The effect of the resonances is given by the second term on the right hand side of Eq. (7). It exhibits a resonance enhancement near the resonance energy $E_{r}$ described by a Lorentzian peak. The width, $\Gamma \approx 2 \pi \rho_{0} v^{2}$, is given by the decay width of individual resonances. Based on physical grounds, we expect that in the limit of weak coupling $(v \ll 1)$ there will be a strong degeneracy due to the resonance states. This degeneracy is responsible for the resonance enhancement of the DOS at $E=E_{r}$. Gradually, as the coupling with the other states is increased, the degeneracy and consequently the peak in $\rho\left(E_{r}\right)$ decrease. These expectations 
are in full agreement with Eq. (7). The same result has been found in the extended RMT, Ref. [6], with the use of the super-symmetry formalism. Here however Eq. (7) appears natural under the frame of the tight-binding picture.

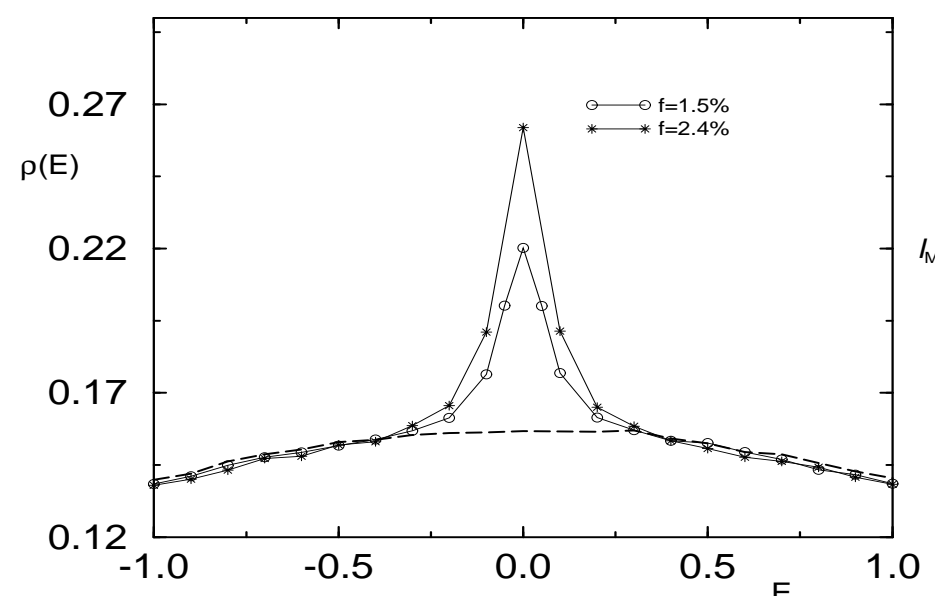

FIG. 2. Density of states $\rho(E)$ for the $2 \mathrm{~d}-$ Anderson model in the presence of resonances with resonance energy $E_{r}=0$, coupling constant $v=0.2$ and various concentrations. The thick dashed line corresponds to the Anderson model without resonances. In all cases the disorder strength is $W=4$.

To verify the validity of the approximations used in the derivation of Eq. (7) and to go beyond the weak coupling limit, we have performed numerical simulations of the 2 d-Anderson model with $m$ randomly distributed resonant states. Our main concern in this study is to investigate how the DOS and the localization length behave as a function of energy for various resonance concentration $f=m /(N+m)$ and coupling strengths $v$. To evaluate these quantities, we used the iterative procedure developed in [10]. This method is particularly suitable for the calculation of various quantities for a macroscopically large system described by a microscopic Hamiltonian. The system we considered is a cylinder of width $M$ in $y$ direction and length $L$ in $x$ direction. For $L$ large compared to $M$ the sample is essentially a quasione-dimensional system. For the above calculations, we have used samples of length $L=10^{5}$ and width up to $M=100$.

We first examine the behavior of $\rho(E)$ as a function of the coupling constant $v$ and the resonance concentration $f$. Our numerical results for low resonance concentration and various values of the coupling $v$ are reported in Fig. 1. As we can see, at $E=E_{r}$ the DOS shows a resonance enhancement which is very well reproduced by the analytical formula (7). As the resonance coupling $v$ increases we observe a gradual decrease of the hight of the Lorentzian peak as well as an increase of its width. In the limit $v=1$ we recover the Anderson case. We also investigated the dependence of the DOS on the concentration $f$ of resonances in the disordered sample. In the weak coupling limit, we found that by increasing the concentration $f$ the Lorentzian peak at the DOS increases in high (see Fig. 2) in agreement with the analytical formula (7). Our numerical calculations, for small resonance coupling $v=0.2$, are presented in Fig. 2. for two particular values of concentration. We remind that exactly the same behavior of $\rho(E)$ was observed for the one-dimensional case [9].

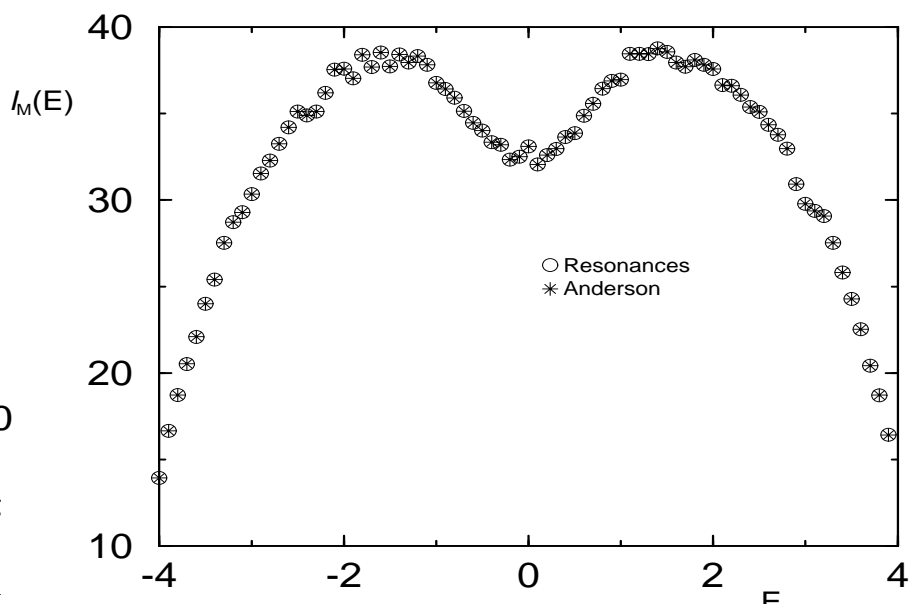

FIG. 3. Localization length $l_{M}(E)$ in the presence (o) and absence $(*)$ of resonances calculated numerically for the $2 \mathrm{~d}-$ Anderson model as a function of energy. The width of the strip is $M=20$, and the disorder strength is $W=4$. The resonance energy is $E_{r}=0$, while the resonance concentration and coupling are $f=2.4$ and $v=0.2$ respectively. It is clear that $l_{M}(E)$ is not affected by the presence of resonances.

Next, we calculate the localization length $l_{\infty}(W, E)$, needed for the determination of diffusion constant $D(E)$. Using the same numerical approach [10 we define a localization length $l_{M}(W, E)$ depending on $M, W$ and $E$. The asymptotic localization length $l_{\infty}(W, E)$ corresponding to the infinite system, is then defined in the limit of $M \rightarrow \infty$ i.e.

$$
l_{\infty}(W, E) \equiv l_{M \rightarrow \infty}(W, E)
$$

The numerical results, performed for different values of resonance concentration, show that $l_{M}(E)$ can be considered approximately independent from the concentration $f$ and from the resonance coupling $v$ (as long as the concentration is not extremely large). Our results remain practically the same for all values of $M$ we have considered in our calculations (up to $M=100$ ) and $f$. In Fig. 3 we present the behavior of the localization length $l_{M}(E)$, for one particular value of $f$, vs. the corresponding one of the standard Anderson model. This behavior of the localization length is totally different from the one found in the one-dimensional case where $l_{\infty}(E)$ decreases drastically everywhere except close to the resonance $E_{r}$ where it is approximately equal to the corresponding one 
obtained for the standard Anderson model [9]. The underline physical reason is that the electron in the twodimensional geometry can bypass the resonances following a different path. We expect that the same behavior will appear also in three-dimensions. In contrast, in the one-dimensional case the electron has to pass through the resonance and thus to suffer resonance tunneling. Thus, the one dimensional structure acts as a filter allowing only electrons with energy $E_{r}$ to transmit.

We now turn our attention to the calculation of the diffusion constant which will be the linking observable with the experimental results [4]. In the quasi-one dimensional multichannel case the diffusive constant $D(E)$ is related with $l_{\infty}(E)$ and $\rho(E)$ through the relation [11]

$$
D(E)=\frac{l_{\infty}(E)}{4 \pi \rho(E)} .
$$

Then, it becomes clear from the previous analysis that $D(E)$ will show a dip near the resonance energy $E_{r}$ (see also [12]), due to the resonance enhancement of the density of states $\rho(E)$. However, in order to compare the behavior of $D(E)$ with the experimental results [4] we have in addition, to take into account the fact that the strength of the resonance coupling $v$ depend on the concentration of resonances $f$ as $v \sim f^{5 / 6}[6]$. Fig. 4 shows $D(E)$ vs. energy for different concentrations. In agreement with the experimental results 刲, we find that an increase of $f$ causes a decrease of $D$ far away from the resonance while the dip near the resonance energy $E_{r}$ widens and eventually disappears for $v \sim 1$. This result identifies the resonant behavior of the DOS as the main explanation for the dip in the diffusion constant. Moreover, it establishes the equivalence of transport properties in the presence of resonances, between classical and quantum waves.

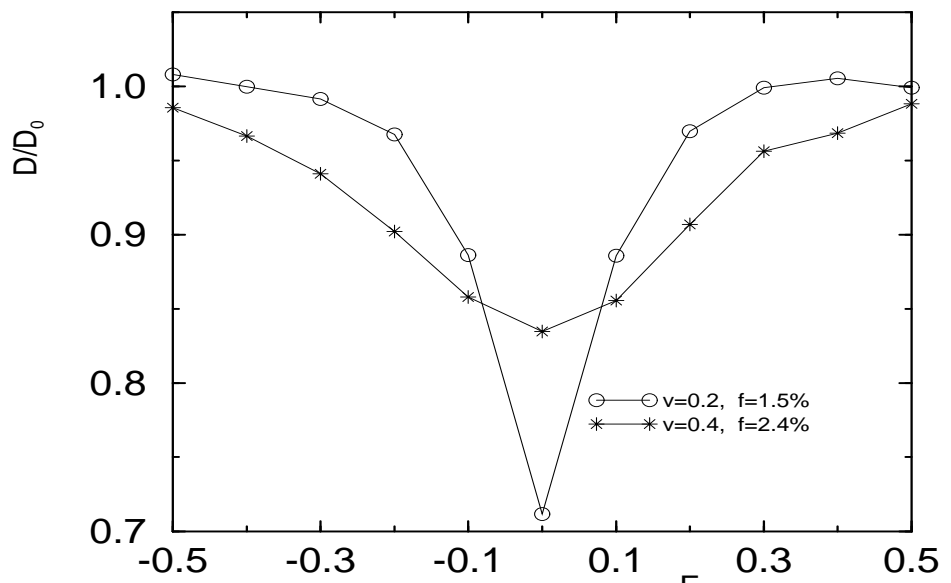

FIG. 4. Normalized diffusion constant $D(E) / D_{0}(E)$ calculated numerically for the 2D-Anderson model as a function of energy. The different curves correspond to different concentrations $f$ of the resonances ( $D_{0}$ is the diffusion constant in the absence of resonances).
In conclusion, we have studied, both analytically and numerically, the effect of resonances on the transport properties of electrons in two-dimensional tightbinding Anderson model. At low concentration and weak coupling the DOS is affected strongly by exhibiting a Lorentzian peak at resonance energy. As the coupling strength increases, the width $\Gamma$ of the Lorentzian increases and the resonance structure in DOS is smeared out. Contrary, the localization length is unaffected from the resonances. An application of the formula, $l_{\infty}(E)=$ $4 \pi \rho(E) D(E)$ 11, identifies the behavior of $\rho(E)$ as the explanation for the observed experimental results of the diffusion constant. This establishes the analogy between classical and quantum waves. Our results are similar to those obtained within the ERMT [6].

Acknowledgements We appreciate discussion with Y. Imry and C. Tejedor. B. E gratefully acknowledge the support of the European Union through the Training and Mobility of Researchers Ultrafast Network .

[1] For a review, see Scattering and Localization of Classical Waves in Random Media, edited by Ping Sheng (World Scientific, Singapore, 1990).

[2] Y. Imry, Introduction to Mesoscopic Physics, (Oxford University Press, New York, 1997).

[3] T. Guhr, A. Muller-Groeling, H. Weidenmüller, Phys. Reports 299 (1998) 189.

[4] Van Albada M., Van Tiggelen B. A., Lagendijk A. and Tip A., Phys. Rev. Lett. 66 (1991); see also N. Garcia, A. Z. Genack, and A. A. Lisyansky, Phys. Rev. B 46, 14475 (1992)

[5] C. M. Soukoulis, S.Datta, and E. N. Economou, Phys. Rev. B 49, 3800 (1994); K. Busch. C. M. Soukoulis and E. N. Economou, Phys. Rev. B 50, 93 (1994).

[6] B. Elattari, V. Kagalovsky, and H.A. Weidenmüller, Phys. Rev. B 5711258 (1998).

[7] see e.g. M. L. Mehta, Random Matrices, 2nd ed. (Academic Press, New York, 1991), and references therein.

[8] B. Elattari, V. Kagalovsky, and H.A. Weidenmüller, Phys. Rev. E, 572733 (1998); B. Elattari, V. Kagalovsky, and H.A. Weidenmüller, Europhys. lett., 4213 (1998).

[9] Brahim Elattari and Tsampikos Kottos, Phys. Rev B 59, R5265 (1999).

[10] A. MacKinnon, B. Kramer, Z. Phys. B-Condensed Matter 53, 1 (1983); A. MacKinnon, Z. Phys. B-Condensed Matter 59, 385 (1985).

[11] K. B. Efetov, Adv. Phys. 32, 53 (1983).

[12] D. Livdan and A. A. Lisyansky, Phys. Rev. B 53, 14843 (1983). 